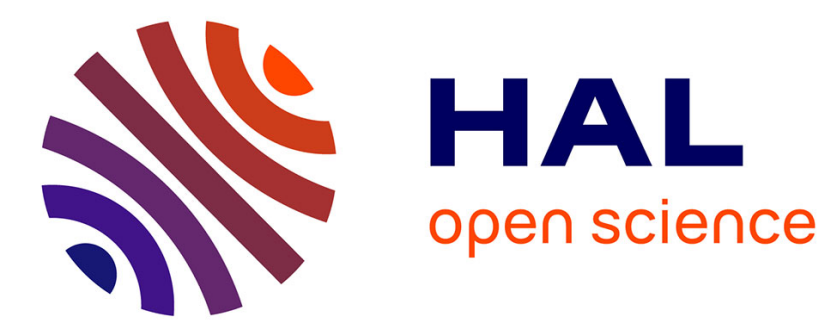

\title{
Les services environnementaux dans les espaces ruraux: une approche par l'économie des services
}

\author{
Olivier Aznar, P. Perrier Cornet
}

\section{To cite this version:}

Olivier Aznar, P. Perrier Cornet. Les services environnementaux dans les espaces ruraux: une approche par l'économie des services. Economie Rurale, 2003, 273-274, p. 142 - p. 157 . hal-00456175

\section{HAL Id: hal-00456175 \\ https://hal.science/hal-00456175}

Submitted on 12 Feb 2010

HAL is a multi-disciplinary open access archive for the deposit and dissemination of scientific research documents, whether they are published or not. The documents may come from teaching and research institutions in France or abroad, or from public or private research centers.
L'archive ouverte pluridisciplinaire HAL, est destinée au dépôt et à la diffusion de documents scientifiques de niveau recherche, publiés ou non, émanant des établissements d'enseignement et de recherche français ou étrangers, des laboratoires publics ou privés. 


\title{
Les services environnementaux dans les espaces ruraux : une approche par l'économie des services
}

Publié dans « Economie Rurale », $n^{\circ}$ 273-274, janvier-avril 2003, p. 142-157.

\author{
Olivier Aznar ${ }^{*}$ et Philippe Perrier-Cornet ${ }^{\#}$ \\ Cemagref Clermont-Ferrand et UMR Inra-Enesad Dijon
}

Dans les débats actuels sur la multifonctionnalité de l'agriculture, la notion de service environnemental est fréquemment employée pour qualifier les différentes activités ou opérations qui contribuent à l'entretien, la préservation, l'amélioration de l'environnement dans ses diverses dimensions (paysage, ressources naturelles, écosystèmes...). Une place centrale est accordée à l'agriculture et aux agriculteurs dans la fourniture de ces services.

Le terme de service environnemental est aussi utilisé dans d'autres débats, dans des acceptions apparemment différentes. C'est par exemple le cas dans le schéma des services collectifs des espaces naturels et ruraux (dans le cadre de la Loi d'orientation pour l'aménagement et le développement durable du territoire du 25 juin 1999). A ces différentes utilisations de la même notion de service, correspondent en fait des processus ou des mécanismes économiques distincts. L'utilisation assez systématique et indifférenciée du terme de service environnemental pose ainsi problème du point de vue de l'analyse économique. La première partie de ce texte est consacrée à une revue de la littérature économique sur ce sujet, qui permet de mettre en évidence l'usage polysémique de la notion de service environnemental; nous proposons d'ordonner cette diversité autour de trois grandes conceptions économiques. Cette mise à plat n'a pas qu'un intérêt théorique, elle peut aussi contribuer à clarifier l'action publique. Dans une deuxième partie, nous proposons une analyse des services environnementaux à partir de l'une des trois démarches passées en revue précédemment, celle de l'économie des services. Cette orientation a l'intérêt de se dégager de l'assimilation qui est faîte trop hâtivement à notre sens entre service environnemental et externalité positive de production. Elle conduit de ce fait à ne pas se focaliser sur les seuls agriculteurs comme producteurs de services environnementaux. Nous l'appliquons ensuite dans le cadre d'une étude concrète sur les services environnementaux mis en œuvre dans trois territoires ruraux en Auvergne. L'étude met en évidence la diversité des logiques d'action des différents prestataires de services environnementaux et relativise la place des agriculteurs dans leur fourniture.

\section{Trois conceptions du service environnemental dans la littérature économique}

\section{Le service environnemental comme produit du capital naturel}

Cette approche renvoie à grands traits au courant de l'économie écologique, qui s'est principalement développée dans les pays anglo-saxons et scandinaves, en particulier autour de la revue Ecological economics. Les services environnementaux y sont définis comme des services rendus par la nature à l'homme, non produits par celui-ci. Ils sont «fournis par le système naturel à la société humaine » (Hannon, 1998).

\footnotetext{
* Olivier.aznar@cemagref.fr

\# Philippe.Perrier-Cornet@enesad.inra.fr
} 
Fondamentalement, l'approche repose sur la notion de capital naturel. « Le capital naturel représente la valeur des réserves de ressources naturelles d'un pays : ressources halieutiques, forêts, gisements miniers, eau et cadre naturel, notamment. Le capital naturel produit des biens et des services exactement comme la population active et le capital matériel » (Gillis et al., 1998, p.229). Les sociétés humaines puisent dans ce stock de capital naturel pour assurer leur reproduction. En tant que stock, celui-ci est la source d'un flux de services qui deviennent source de valeur pour les hommes. De plus, les services environnementaux produits fournissent les fonctions de maintien de la vie nécessaires à la reproduction du capital naturel lui-même (Collados et al., 1999).

Pour certains tenants de l'économie écologique, les services environnementaux eux-mêmes font partie du capital naturel. C'est ce qui ressort de la définition de Berkes et Folke (1992) pour qui le capital naturel recouvre l'ensemble des ressources naturelles ainsi que l'ensemble des « services environnementaux tels que le maintien de la qualité de l'atmosphère, le climat, le fonctionnement du cycle hydrologique incluant le contrôle de la marée et la fourniture d'eau potable, l'assimilation des déchets, le recyclage des nutriments, la création de sols, la pollinisation des cultures, la fourniture de nourriture par la mer et le maintien d'un vaste patrimoine génétique » (ib., p. 2) assurés gratuitement par les écosystèmes et la biosphère qui permettent la constitution et le maintien d'un milieu de vie.

Costanza et al. (1997) proposent une taxonomie complète de ces services environnementaux (également dénommés « ecosystem services »). Ils les classent en 17 grandes catégories qui recouvrent un large spectre : régulation de la composition de l'air (balance $\mathrm{CO}_{2} / \mathrm{O}_{2}$, fourniture d'ozone pour la protection UV....), fourniture et régulation de l'eau, pollinisation, fourniture de nourriture, mais aussi fourniture d'opportunités pour des activités récréatives de pleine nature... Dans leurs analyses, la fourniture des services du capital naturel est souvent combinée (mais pas systématiquement) avec celle des services fournis par le capital humain ou matériel.

Une des questions essentielles pour l'économie écologique est de parvenir à traiter dans l'analyse économique le capital naturel au même titre que le capital matériel et le capital humain. Ainsi, dans cette acception, le service est identifié à l'effet utile comme dans la théorie néo-classique d'inspiration walrasienne en général. L'économie écologique pose d'abord la question de l'évaluation de la valeur du capital naturel et de l'évolution de celle-ci (comme les autres formes de capital, il peut se dégrader ou augmenter de valeur et on doit pouvoir lui appliquer des raisonnements en termes d'investissement et d'amortissement du capital). Les services environnementaux jouent un rôle important dans ce débat, puisque selon certains auteurs partie prenante de cette approche, une façon de fixer la valeur du capital naturel consiste à estimer les bénéfices nets tirés de la production des services environnementaux fournis à la société à partir de ce capital. Un autre débat important dans ce courant concernant les services environnementaux porte sur les substituabilités et complémentarités entre capital naturel et «capital créé par l'homme» et les modalités de celles-ci. Par ailleurs, l'économie écologique, en mettant exclusivement l'accent sur les services environnementaux fournis par le capital naturel, ne prend pas en compte que le capital matériel peut lui-même être à la source de services environnementaux, par exemple de correction d'externalités négatives. D'une façon générale, nous ne nous inscrivons pas dans cette perspective du service environnemental, qui repose sur la notion d'effet utile, dans la suite de notre travail.

Lorsqu'ils parlent de «services d'environnement fournis par la forêt» (et non par la sylviculture ou les sylviculteurs), A. Desprès et D. Normandin (1996) sont de fait assez proches de cette conception, bien qu'ils considèrent dans le même temps que ces services sont des externalités et des biens publics dont la «qualité résulte de l'activité de production de 
certains agents [qui] bénéficient sans échange monétaire à d'autres agents » (1996, p.69). Mais ces mécanismes économiques qu'ils invoquent renvoient plutôt à un autre corpus d'analyse économique, celui de l'économie de l'environnement.

\section{Le service environnemental comme externalité positive de production}

Alors que l'approche précédente du service environnemental ressort du domaine de l'économie des ressources naturelles, celle qui relève de l'économie de l'environnement considère de fait le service environnemental comme une externalité positive de production (cf. encadré).

Rappelons qu'il y a externalité positive de production lorsque les actions d'une ou plusieurs firmes bénéficient à d'autres agents (producteurs ou consommateurs) sans que la/les firme(s) émettrice ne reçoive de compensation financière pour les bénéfices engendrés pour les autres, ni que les bénéficiaires de cet effet externe n'aient à débourser pour cet effet dont ils bénéficient. Par exemple, « un maillage de type bocager améliore la productivité des vaches laitières et engendre des effets externes au bénéfice des consommateurs (aménités pour le promeneur, protection de la faune) et des firmes (...inputs pour le tourisme) » (Bonnieux, Desaigues, 1998, p.21). Ces effets environnementaux positifs de l'activité économique (et non de l'activité de la nature elle-même) qui procurent aux individus un bien être plus élevé et/ou aux firmes un profit extra sont qualifiés par de nombreux auteurs de "services environnementaux » (par ex. Madelin, 1995) ou de «biens et services environnementaux » (Mahé, 2001), sans que soit d'ailleurs explicitée la distinction éventuelle faite entre biens et services dans ce type d'approche. Les travaux de l'OCDE sur les aménités rurales (OCDE, 1994) et sur la multifonctionnalité de l'agriculture (OCDE, 2001) s'inscrivent également dans cette conception.

Les externalités

Le concept d'externalité est très simple à comprendre en première approche, tout en étant employé dans des sens très divers. En économie de l'environnement, la plupart des auteurs retiennent la définition formulée par Baumol et Oates qui repose sur deux conditions, dont on ne retient en général que la première comme définition : « une externalité est présente chaque fois que des fonctions d'utilité ou de production d'un individu (appelé A) contiennent des variables réelles (c.à.d. non monétaires) dont les valeurs sont choisies par d'autres (personnes, firmes, gouvernements) sans attention particulière aux effets sur le bien être de A » (Baumol et Oates, 1988). Baumol et Oates soulignent de plus ce point essentiel à nos yeux : « la définition exclut les cas dans lesquels quelqu'un fait délibérément [souligné par les auteurs] quelque chose pour affecter le bien être de A » (ib., p. 17). Cornes et Sandler (1996) développent aussi cette conception non intentionnelle de l'externalité lorsqu'ils écrivent que la théorie des biens publics et des externalités offrent les fondations de l'économie de l'environnement «pour laquelle les activités économiques sont comprises comme produisant des effets secondaires environnementaux souvent ignorés par celui qui les a générés » (ib. p. 12). De la même façon, Dorfman (1993) insiste sur le fait que les externalités sont involontaires. Pour cet auteur, les externalités ne peuvent être ni des activités bénévoles, ni des agressions envers d'autres personnes.

L'assimilation entre externalité positive de production et service environnemental pose à notre sens quatre questions.

Tout d'abord, s'agissant d'externalités positives de production, leur fourniture va être, pour l'essentiel, le fait d'agents utilisant des éléments d'environnement, des ressources naturelles dans leur combinaison productive (le sol en premier lieu) : les agriculteurs, et secondairement les sylviculteurs, sont ici implicitement considérés comme les principaux producteurs de services environnementaux ainsi définis et ceux-ci sont des sous-produits d'une production jointe (agricole, sylvicole). L'essentiel de la littérature et des travaux empiriques d'économie sur les biens et services environnementaux menés dans ce cadre d'analyse est focalisé sur les 
agriculteurs, même si certains travaux s'intéressent plus particulièrement aux relations entre les services environnementaux et le tourisme rural (Le Goffe et Delache, 1997).

Ensuite, dans ce cadre, stricto sensu la fourniture du service environnemental est non intentionnelle. En soi, que l'activité agricole de l'agriculteur engendre ou non des externalités positives sur l'environnement ne modifie pas a priori la structure des coûts : il n'y a ni intentionnalité, ni coût spécifique pour le service environnemental rendu s'il s'agit bien d'un effet externe. En ce sens, elle ne donne en général pas lieu à un coût de production supplémentaire par rapport à celui de l'activité principale de l'émetteur. Bonnieux et Rainelli (2000) l'illustrent bien dans leur analyse des différentes liaisons possibles entre la production de denrées agricoles et la fourniture d'aménités : « ... la complémentarité qui prévalait [entre production de biens agricoles et de biens environnementaux] est dite faible, dans la mesure où la fourniture d'aménités n'a pas un caractère intentionnel et leur coût marginal de production est proche de zéro » (ibid., p. 808).

Puis, sur un plan normatif, l'approche de l'économie de l'environnement conduit à rechercher les solutions pour que les effets/services environnementaux de l'activité économique des agriculteurs procurent aux usagers finaux le bien être le plus élevé. Les externalités environnementales doivent être internalisées, des signaux doivent être transmis à l'émetteur au niveau de demande formulée par le récepteur du service environnemental : les producteurs de ces services doivent être incités à les fournir et être rémunérés pour cela à hauteur de l'avantage marginal social procuré par le bien ou service environnemental. Mais ces services ont un caractère de biens publics ou quasi-publics (Mahé et Ortalo-Magné, 2001) accessibles à tous, sans rivalité : faute de marché, on ne peut pas en général faire payer leurs utilisateurs et c'est à la puissance publique de subventionner la fourniture de ceux-ci pour garantir qu'ils soient fournis au niveau attendu par la société. Les auteurs recourent alors à des méthodes d'évaluation économiques de l'environnement, censés mesurer l'avantage social lié aux externalités positives. Ces méthodes relèvent de trois approches ${ }^{1}$ principales : la méthode des coûts de déplacement, celle des prix hédonistes et celle d'évaluation contingente. Cette dernière, même si elle suscite des controverses (Willinger, 1996), a en particulier été appliquée aux externalités positives de production (par exemple : Dabbert et al, 1998).

Enfin, l'économie de l'environnement permet de traiter des externalités positives, mais aussi des externalités négatives. Ces dernières ne sont pas entendues comme des services environnementaux par les économistes de l'environnement, car elles sont la source de déséconomies pour les usagers finaux (sur l'analyse des pollutions, cf Bonnieux et Desaigues, 1998). En revanche, les dépenses volontaires pour corriger des externalités négatives peuvent être considérées comme des services environnementaux, mais selon une définition différente, qui relevera de l'économie des services (cf. infra).

Dans une approche en termes de service-externalité, la question de l'internalisation, de la rémunération de l'émetteur devrait se faire en regard de l'avantage marginal social procuré par le service environnemental, en fonction de la valeur des avantages fournis aux utilisateurs. Elle ne devrait pas se faire en référence à la rémunération du coût de production du service, de l'effort spécifique fourni par son émetteur, puisque par définition, celui-ci est nul et que la production du service n'est pas intentionnelle. Or, ce point n'est pas toujours clair dans les travaux d'économie de l'environnement appliqués aux biens et services environnementaux, puisque ceux-ci développent dans le cadre d'un raisonnement conduit en terme d'externalités l'idée d'une rémunération «en rapport avec le travail accompli par les agriculteurs » (Mahé, 2001) ou « en compensation d'un effort particulier contribuant à une meilleure prise en compte de l'environnement » (Madelin, 1995, p.77). Il nous semble que dans ce cas, on ne peut plus se cantonner au seul champ de l'économie de l'environnement. La démarche se

1. Ces méthodes sont décrites en détail par Desaigues et Point (1993) ou Bonnieux et Desaigues (1998) 
rapproche alors de celle de l'économie des services, la question est alors de savoir comment, un service est produit ou fourni d'une façon intentionnelle en réponse à quelle demande d'intervention.

\section{Le service environnemental dans le champ de l'économie des services}

L'économie des services rassemble des travaux très hétérogènes, avec cependant un élément commun qui traverse toutes les approches développées en économie des services : le débat sur la distinction à opérer entre les biens et les services. Ce débat, qu'il est hors de propos de reprendre ici, a fortement évolué, en particulier dans la période contemporaine, alors que, historiquement, depuis les physiocrates et A. Smith il a été longtemps focalisé sur la frontière entre les activités productives et celles considérées comme improductives.

Dans une première acception, peu théorisée, tout un ensemble de travaux se référant à l'économie des services partent d'une délimitation des activités de services en termes de secteurs d'activité (i.e. toutes les activités ne pouvant être classées dans le secteur primaire ou le secteur secondaire) sans que cette délimitation ne fasse vraiment l'objet d'un travail de définition théorique. En référence à ce cadre, des délimitations des services environnementaux ont été produites, par exemple celle actuellement utilisée dans les négociations internationales de l'Organisation Mondiale du Commerce (OMC, 2000) ou par l'Organisation des Nations Unies (CNUCED, 1998). Sept classes de services environnementaux sont catégorisées dans les dossiers de l'OMC : eau destinée à la consommation humaine et gestion des eaux usées, gestion des déchets, protection de l'air et du climat, remise en état et nettoyage du sol et de l'eau, lutte contre le bruit, protection de la diversité biologique et des paysages, autres services environnementaux. L'attention dans cette approche est focalisée sur les secteurs et les entreprises qui agissent dans ces domaines de l'environnement : assainissement, gestion des déchets, distribution et recyclage de l'eau... Les grands opérateurs dans ces secteurs y occupent une place importante: «Nous ne sommes pas des marchands d'eau. Nous ne vendons pas un produit mais assurons un service, celui de rendre disponible à chacun et en permanence une eau purifiée et de restituer cette eau au milieu naturel après traitement. C'est le prix de ce service qui est facturé et non l'eau comme matière première » (G. Mestrallet, PDG du groupe Suez in Le Monde, 26 oct. 2001).

Parallèlement à ces approches, une réflexion théorique sur l'économie des services est menée depuis une vingtaine d'années, suite aux travaux fondateurs de Hill (1977). En France, Delaunay et Gadrey (1987) ont été les premiers à proposer une définition positive des services, s'inspirant en partie de celle de Hill. Fondamentalement, cette approche envisage le service comme un rapport social particulier entre la production et l'usage, .

Delaunay et Gadrey (1987, p. 212) proposent la définition suivante : on pourrait énoncer qu' « il y a service lorsqu'une unité (ou agent) économique ou institutionnelle $\mathrm{B}$ fait appel à une autre unité (ou agent) A, pour effectuer, pour le compte de B, une opération sur un point d'application $\mathrm{C}$ appartenant à $\mathrm{B}$ (ou dont les transformations relèvent de la responsabilité sociale de $B$ ), opération dont le résultat immédiat sur $C$ n'est pas séparable de $C$, et ne peut donc circuler économiquement de façon autonome ». Ce support $\mathrm{C}$ peut être un objet, un bien, un individu (qui peut être confondu avec l'usager ou distinct de lui), un flux ou un stock d'informations ou encore une organisation, mais il reste toujours indissociable de la prestation de service. Cette définition est habituellement représentée par le schéma du «triangle de service $»$, (cf. ci-dessous, fig.1). 
Figure 1 : le triangle de service

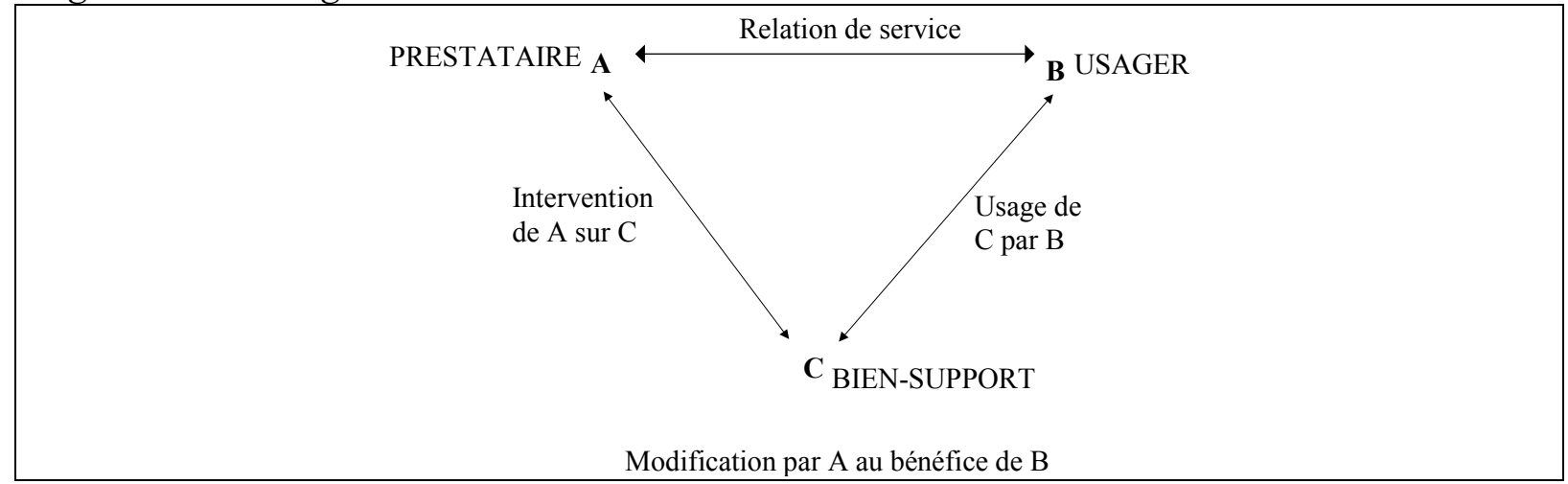

Delaunay et Gadrey (1987) insistent sur le fait que la relation de service ne se réduit pas à la dimension interpersonnelle. D'une part, elle nécessite l'utilisation de capital (inputs et moyen de production) (Delaunay, 1999). D'autre part, elle engage des institutions extérieures à la relation stricto sensu dans un rapport social de service. Ce n'est donc pas la dimension relationnelle qui caractérisent la production de service - c'est également vrai de la production industrielle - mais bien la façon dont circule économiquement le produit du service.

Gadrey a depuis repris et fait évoluer cette définition, en développant trois logiques de recours caractérisant les activités de service : l'intervention, la mise à disposition et la logique de spectacle vivant (Gadrey, 2000). D'une façon générale, il y lieu de souligner dans cette approche de l'économie des services qu'un service est toujours une relation orientée, conçue de façon intentionnelle, en vue de permettre un usage d'un support par un (ou plusieurs) usager(s).

Une autre dimension importante sur laquelle insistent certains auteurs d'économies des services concerne la coproduction entre prestataire et usager du service (de Bandt, 1995). Elle signifie que l'usager joue un rôle important dans la production pour " révéler » ses besoins et produire des informations pertinentes pour le prestataire. La propriété de coproduction ne suffit pas à distinguer la production d'un service de celle d'un bien, mais elle est souvent présente dans les services (à des degrés variables).

Cette démarche d'économie des services a jusqu'à maintenant été très peu utilisée pour l'analyse des services environnementaux. C. Laurent a tenté de l'appliquer dans le domaine de la production du paysage rural (Laurent, 1994). Dans son analyse, la "réalité C » est le territoire, le prestataire A est l'agriculteur, l'usager B est la collectivité. Mais son analyse reste focalisée sur l'intervention des agriculteurs sur le territoire et ne s'intéresse pas à d'autres prestataires éventuels de services environnementaux, alors que l'entrée par l'économie des services permet de sortir de cette focalisation. Cette dernière approche offre la possibilité de traiter de support dont la valeur ne circule pas économiquement, ce qui est le cas des biens de nature hors marché.

Notre objectif est de repartir de ce type d'approche d'économie des services développée par Hill, Delaunay et Gadrey, et de la mettre en œuvre dans le cas des services environnementaux, sans nous limiter a priori à une catégorie particulière de prestataires -les agriculteurs-, mais au contraire en l'utilisant pour, entre autres, situer la place des agriculteurs dans l'ensemble de ce champ des services environnementaux. C'est en effet, à notre sens, l'un des intérêts du passage par l'économie des services. 


\section{Une lecture des services environnementaux du point de vue de l'économie des services}

\section{Définition des « services » environnementaux}

En prenant comme référence générale le triangle de service, le service environnemental peut être défini comme une intervention sur un bien de nature (non marchand), en vue de permettre l'usage environnemental de ce dernier. Les attributs environnementaux du bien-support sont améliorés ou maintenus en l'état.

La définition retenue pour le service environnemental s'appuie également sur le caractère intentionnel de la fourniture du service : l'amélioration de l'environnement est intentionnelle (ou délibérée) du point de vue du prestataire et elle répond à une demande collective relative à un support non marchand. Le caractère intentionnel d'une action peut se traduire économiquement en termes de temps et/ou d'argent consacrés à la fourniture du service environnemental. Le prestataire y consacre un temps spécifique, en échange d'une rémunération par les usagers, ou, plus souvent, par un commanditaire agissant au nom des usagers.

En effet, du fait du caractère de bien public des biens de nature, les usagers des services environnementaux mis en œuvre sur ces biens ne sont pas en général ceux qui formulent directement (et prennent en charge) la demande de service auprès des prestataires. C'est le cas extrême pour des services visant à la protection des ressources ou la conservation de la biodiversité dont les usagers seront les générations futures. Si les usagers ne participent pas directement à la relation de service, d'autres agents agissent en leur nom. Ces commanditaires, qui prennent place entre le prestataire et l'usager et formulent la demande de service auprès des prestataires au nom des usagers, jouent un rôle central dans le cas des services environnementaux à dimension publique. Ce sont aussi eux qui rémunèrent la prestation. Ces commanditaires ont le plus souvent un caractère d'agent public. Dans le cas de services environnementaux privés, d'autres types d'intermédiaires peuvent également exister, à l'instar des catégories générales de services (Gadrey, 1994) : ils exercent des fonctions diverses lors du déroulement du service: évaluation, édiction de normes, règlement de litiges... Mais il s'agit d'une autre catégorie d'intermédiation, qui ne va pas aussi loin dans la substitution à l'usager pour la commande du service.

\section{Classification}

Nous classons l'ensemble des activités ou actions qui contribuent -intentionnellement ou nonà l'amélioration, l'entretien, la restauration etc. des biens de nature, en trois grandes catégories, dont seule la première relève pleinement de la notion de service, du point de vue de l'économie des services :

Les services environnementaux complets (au sens de l'économie des services)

Ils correspondent aux services qui comportent l'ensemble des pôles du triangle. La production est intentionnelle, elle répond à une demande portée par un commanditaire. Le prestataire est rémunéré pour une intervention sur un bien-support de nature, le coût de production du service est non nul. Le service environnemental complet se caractérise également par une coproduction entre le prestataire et le commanditaire qui précise les attendus du service.

Ce service environnemental peut être exclusif ou joint si sa fourniture par le prestataire est associée à une autre activité. Mais dans ce cas, il y a bien un effort spécifique et un coût supplémentaire pour la fourniture du service environnemental, en plus de celui de l'activité jointe. 


\section{Les externalités positives de production}

Il y a un effet positif sur l'environnement d'une activité d'un agent économique, mais sans intentionnalité ni coût supplémentaire. Cette catégorie ne relève pas de l'économie des services. Dans le cas d'un service, l'accord entre le prestataire et l'usager est essentiel, car sans lui, les changements correspondraient à des services non sollicités (Hill, 1977). L'amélioration de l'environnement n'est pas intentionnelle pour l'émetteur de l'externalité, elle constitue en quelque sorte une production fatale (au sens de la comptabilité nationale) induite par une production principale, et donc sans coût de production supplémentaire par rapport à l'activité principale. À l'inverse du service environnemental complet, aucun processus de coproduction ne prend place entre l'émetteur de l'externalité et les usagers finaux de cette dernière.

\section{Les quasi- services environnementaux}

Ils correspondent à des situations intermédiaires où une partie seulement des conditions nécessaires posées pour rentrer dans la catégorie des services est remplie (intentionnalité, coût de production non nul, rémunération du prestataire en réponse à une demande).

Deux cas peuvent être distingués :

- lorsqu'il y a rémunération d'une externalité positive de production, sans qu'il y ait effort spécifique ni coût de production supplémentaire pour le prestataire. Nous désignons cette situation comme un quasi-service environnemental passif. Le terme "passif » renvoie au fait que le prestataire ne fournit pas intentionnellement le service. Par contre, l'intermédiaire le rémunère dans certaines limites définies en fonction de critères ayant trait à l'environnement, même si ce ne sont pas les seuls critères pris en compte. Pour illustration de ce cas de figure, on peut prendre comme exemple les actions d'entretien des prairies conduites par les agriculteurs dans le cadre de la "prime à l'herbe », tout au moins au début de sa mise en place. Ces actions étaient rémunérées par les pouvoirs publics, mais elles n'entraînaient pas de coût de production supplémentaire de la part de l'agriculteur ( $c f$. infra).

- lorsqu'il y a intentionnalité et coût de production, mais pas de rémunération (absence de demande explicitée : le pôle usager-demandeur de l'intervention n'est pas présent). En l'absence d'une véritable demande ou attente sociale, un " service » environnemental peut exister dans la mesure où l'offreur le produit de façon intentionnelle. Nous qualifions cette situation comme un quasi-service environnemental altruiste. Elle correspond au cas où un agent économique adopte un comportement dans le but d'augmenter le bien-être d'autres agents économiques sans qu'il soit, pour autant, rémunéré. Par exemple, une association de pêcheurs entretient les berges d'une rivière, non seulement pour améliorer les postes de pêches, mais les pêcheurs en font aussi « un peu plus » que pour les seuls besoins de loisir (sinon, il ne s'agirait que d'une externalité positive) pour rendre la rivière encore plus agréable aux promeneurs, aux habitants locaux, sans qu'une demande ne leur ait été explicitement formulée.

Le tableau suivant présente une synthèse de ces éléments de classification.

Tableau 1 : Classification des services environnementaux

\begin{tabular}{|l|l|l|}
\cline { 2 - 3 } \multicolumn{1}{l|}{} & $\begin{array}{l}\text { REMUNERATION DU } \\
\text { PRESTATAIRE }\end{array}$ & $\begin{array}{l}\text { COUT DE PRODUCTION VIS-A-VIS } \\
\text { DE L'ENVIRONNEMENT }\end{array}$ \\
\hline SERVICE ENVIRONNEMENTAL COMPLET & oui & oui \\
\hline $\begin{array}{l}\text { QUASI-SERVICE ENVIRONNEMENTAL PASSIF } \\
\text { ALTRUISTE }\end{array}$ & oui & non \\
\hline EXTERNALITE POSITIVE DE PRODUCTION & non & oui \\
\hline
\end{tabular}


Cette classification peut être utilisée dans une vision dynamique des services environnementaux, sans présupposer de trajectoire unique. On la mettra en œuvre plus loin en reprenant le cas de la prime à l'herbe : dans la pratique, les effets positifs sur l'environnement ciblés par ce dispositif ont évolué, passant d'une externalité positive à un quasi-service passif puis, dans certains cas, à un véritable service environnemental fourni par les agriculteurs.

\section{Caractéristiques et spécificités du service environnemental dans le champ de l'économie des services}

\section{a. La nature du bien-support}

Le support du service environnemental est un bien de nature, faisant partie du patrimoine naturel. Si l'on s'inspire de la comptabilité du patrimoine naturel (Comolet, 1994), la catégorie des biens de nature regroupe la faune, la flore, les ressources du sous-sol, ainsi que les milieux physiques non vivants, mais indispensables à la vie (eau, air, sol). Cette définition des biens de nature exclut les éléments artificiels, mais elle retient les éléments ou systèmes naturels aménagés par l'homme, tel un lac artificiel.

En général, l'usage des caractéristiques environnementales d'un bien de nature répond à la caractéristique de non-rivalité. De plus, il est techniquement difficile d'exclure un usager final de la jouissance des caractéristiques environnementales d'un bien de nature. Ainsi, un bien de nature présente une dimension de bien public (ni rivalité, ni exclusion) au sens de Samuelson. Mais il existe de nombreuses exceptions. En effet, souvent, ces biens ne sont pas libres d'accès. Par exemple, les milieux physiques, en particuliers les sols, sont souvent appropriés. De ce fait, on peut exclure certains usagers de leur usage. Ils présentent alors des caractéristiques de biens privés sur le plan économique. De plus, le principe de non rivalité inhérent aux biens de nature peut être partiellement remis en cause. En effet, la présence d'un trop grand nombre d'usagers d'un bien de nature diminue le bien-être global (effets de congestion). C'est le cas par exemple d'un site naturel très fréquenté.

\section{b. La variété des prestataires possibles}

Les services environnementaux peuvent être fournis aussi bien par des entreprises privées spécialisées dans le secteur de l'environnement (entreprises paysagères, de traitement de l'eau ou des déchets etc.) que par des entreprises agricoles pour lesquelles la fourniture d'un service environnemental est couplée, jointe à une autre activité. Un raisonnement analogue peut être mené pour les associations et entreprises d'insertion, présentes dans ce secteur et pour lesquelles la fourniture de services environnementaux est couplée à un objectif d'insertion professionnelle de personnes en difficulté. Vu l'importance des biens publics et en particulier des biens publics localisés en jeu dans la fourniture de services environnementaux, les collectivités locales peuvent être également fortement impliquées comme prestataires de ces services.

Par ailleurs, certains prestataires peuvent être dans une situation spécifique par rapport au bien-support : ils peuvent en effet être eux-mêmes les propriétaires des biens-supports (ou en contrôler l'usage) sur lesquels on leur demande d'intervenir. Ce cas de figure n'est pas commun pour les catégories générales de services, le prestataire intervenant le plus souvent sur un support appartenant à l'usager.

\section{d. La dimension « institutionnelle » de leur production}


Les services environnementaux à caractère collectif n'apparaissent pas ex nihilo. Ils émergent et sont produits dans un cadre institutionnel qui en détermine pour partie la nature et les effets. Pour être réalisés, ils font appel à des circuits de financement divers. Notamment, différentes institutions de régulations, de nature publique ou privée, exercent des fonctions diverses lors du déroulement du service environnemental. Ces institutions, selon le type de financements mobilisé, selon le type d'agent prestataire ou encore selon le type de bien-support peuvent être rattachées à différents secteurs. Nous utilisons la notion de secteur institutionnel pour caractériser le cadre institutionnel dans lequel ces services sont produits. Dans chacun de ces secteurs, la production du service environnemental est faite en référence à des normes, des règles, des conceptions de la qualité différentes.

Ces considérations conduisent à proposer, à partir du triangle de services, un schéma des services environnementaux plus complet (cf. figure 2)

Figure 2 : Représentation schématique des services environnementaux

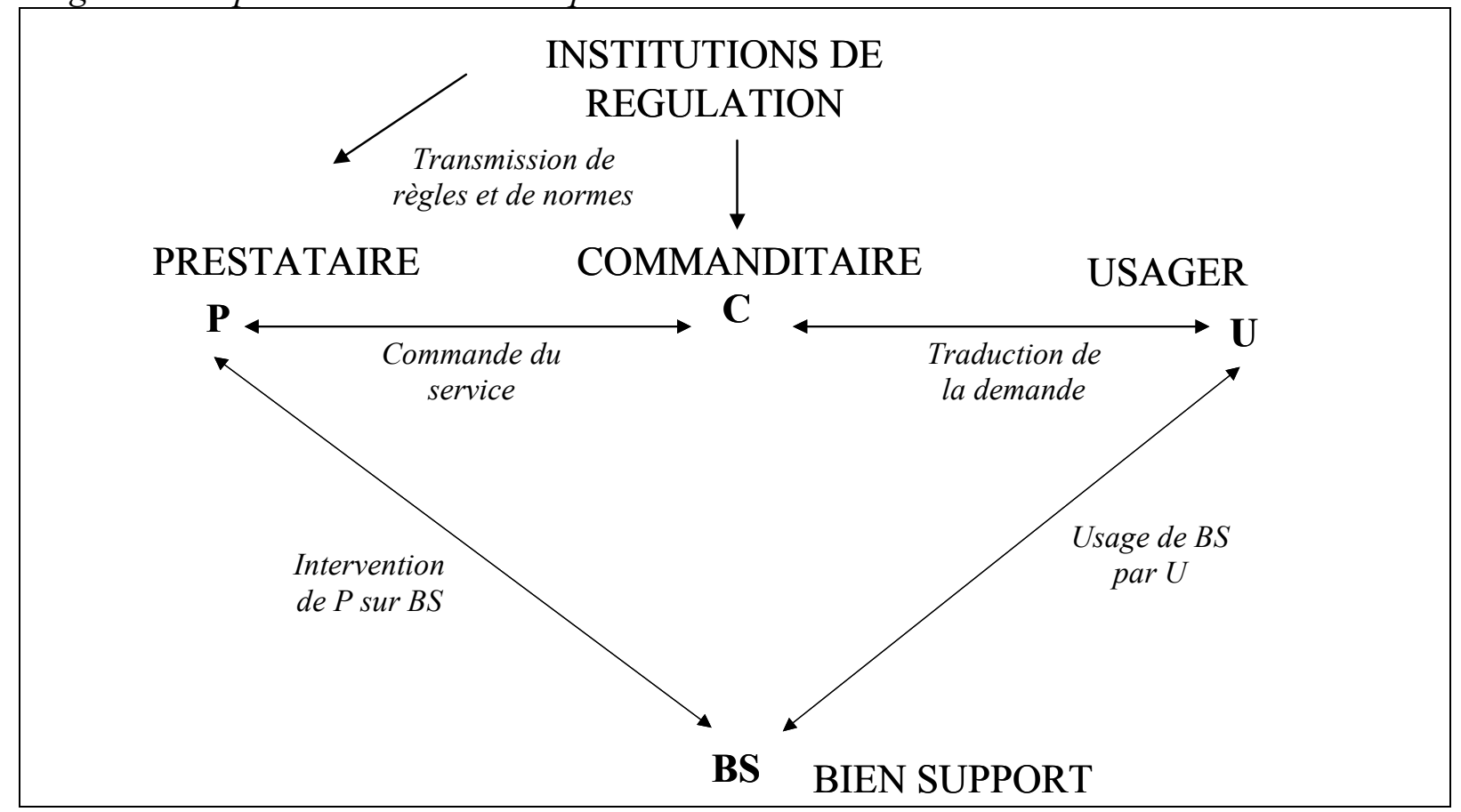

\section{Une analyse concrète des services environnementaux produits dans trois territoires ruraux}

\section{Le champ de l'étude}

Nous nous sommes intéressés dans cette étude aux activités intentionnelles d'entretien, d'amélioration, de transformation de biens-supports environnementaux visibles et ancrés sur le sol (et par définition faiblement artificialisés). Ces biens-supports constituent en quelque sorte les bases matérielles d'un paysage rural. Ils concernent les usages résidentiels et récréatifs actuels des espaces ruraux, c'est-à-dire le cadre de vie des populations résidentes et les activités de pleine nature. N'est donc prise en compte dans cette étude qu'une partie du champ potentiel des services environnementaux, laissant de côté en particulier les 
interventions sur des biens de nature non visibles, celles visant à préserver certaines ressources naturelles ou à prévenir des risques majeurs comme le changement climatique.

L'analyse a été menée sur treize communes rurales d'Auvergne réparties sur trois petites zones ( $c f$. encart méthodologique). A ce niveau géographique fin, elle avait en premier lieu pour objectif de faire un inventaire aussi systématique que possible des «services environnementaux » dans le champ que nous avons retenu, en reprenant notre classification issue de l'économie des services. Nous nous sommes particulièrement attachés à repérer tous les différents types de prestataires et les modalités de production des services. Au delà de l'inventaire statique, l'étude visait également à mettre en évidence en dynamique d'éventuelles trajectoires, à partir des différentes catégories de " services » environnementaux que nous avons construites. Dans le même sens, nous avons essayé de quantifier l'évolution de l'importance des services environnementaux produits par les différents types de prestataires.

D’une façon générale, quatre principales catégories d'opérations de changement d'état peuvent être identifiées pour ces services, selon le degré de transformation du bien-support: les activités d'entretien du bien-support, l'intégration paysagère (concernant un bien-support non naturel situé dans un cadre naturel ), les activités de restauration, l'aménagement (modification des caractéristiques paysagères d'un support en rapport avec un nouvel usage, sans limiter l'usage antérieur). Dans la pratique, les principaux services que nous avons recensés sont les suivants: entretien des berges de cours d'eau, restauration de petit patrimoine, intégration paysagère de bâtiments agricoles, élagage des arbres ornementaux, création ou entretien d'espaces verts, intégration de décharges, aménagement paysager de bourgs, fleurissement, enfouissement de réseaux, aménagement paysager de la voirie, réfection et entretien de parcelles et de leurs bordures (haies), plantation de haies ou d'arbres avec un objectif paysager, déboisement paysager de zones forestières, réaménagement de carrières, pose de franchissement de clôtures pour la randonnée, création et entretien d'espaces d'activités de nature, débroussaillage de chemins et balisage pour la randonnée.

Méthodologie
Les investigations ont été menées sur trois territoires ruraux choisis en Auvergne, étudiés sur la période 1990-
2000 : une zone tournée principalement vers la production agricole (zone de Sancy-Artense) située à l'ouest du
massif du Sancy, une autre essentiellement touristique (zone du Massif du Sancy) et une troisième dominée par
une logique de périurbanisation (zone des Cheires). Chacune des zones, structurée en communauté de
communes, compte une dizaine de communes. L'inventaire des services environnementaux a été mené sur un
ensemble de 3 à 6 communes dans chaque zone d'étude, soit au total 13 communes.
Pour mener à bien cet inventaire, nous avons mobilisé et croisé deux types de sources de données. D'une part,
des entretiens directs dans chaque commune (99 au total) auprès de personnes ressources (élu communal,
agriculteur, membre de l'association de pêche, membre de l'association de chasse, personne d'une association
locale sportive ou environnementaliste...); d'autre part, des entretiens auprès des gestionnaires de procédures
publiques finançant des actions ou opérations ayant un impact sur l'environnement, complétés par un
dépouillement systématique des dossiers administratifs de demande d'aides publiques dans le domaine de ces
" services » environnementaux. Au total, pour les 13 communes étudiées, 641 dossiers ont été dépouillés dans
les domaines des politiques agricoles, des politiques de développement local, des politiques d'emploi, des
politiques des sports et loisirs de plein air et des politiques d'environnement.
Ces deux sources (enquêtes directes et dépouillements des dossiers administratifs) ont été réutilisées et
approfondies pour quelques dispositifs, comme celui de la prime à l'herbe, pour reconstituer l'évolution de la
nature du « service » correspondant à ce financement. Toutes les dépenses sont indiquées en euros courants. Il
n'a pas paru nécessaire de pallier les effets de l'inflation sur la décennie 1990.

Dans le cadre de cette étude, l'analyse du rôle des collectivités locales dans la production de services environnementaux a fait l'objet d'un travail spécifique (Aznar et al., 2002) que l'on ne reprendra pas ici. Soulignons simplement que durant les années 90, les dépenses des 
communes pour les services environnementaux ont très fortement progressé et que celles-ci ont élargi leur champ d'intervention dans le domaine de l'environnement.

\section{L'hétérogénéité de l'offre de services environnementaux}

Notre inventaire a permis de dénombrer 429 services environnementaux complets dans les trois territoires ruraux, sur la période 1990-2000. Leur importance économique est en générale assez faible, bien que relativement hétérogène. Pour $40 \%$ d'entre eux, l'estimation du montant financier des travaux réalisés est inférieure à $3000 €$ pour chaque service. En parallèle, un peu moins de $25 \%$ des services environnementaux complets dépasse $15000 €$. Parmi eux, 14 opérations se détachent avec un coût de plus $150000 €$ pour chacune d'entre elles. Cela correspond à trois types de services environnementaux : la création ou l'entretien d'espaces pour les activités de pleine nature, l'aménagement paysager de bourg et l'enfouissement de réseaux électriques ou téléphoniques.

Tableau 2 : Les prestataires de services environnementaux dans les trois zones

\begin{tabular}{|c|c|c|c|c|c|}
\hline \multirow[t]{2}{*}{ PRESTATAIRES } & \multicolumn{2}{|c|}{ OPERATIONS } & \multicolumn{2}{|c|}{$\begin{array}{l}\text { IMPORTANCE ECONOMIQUE } \\
\text { CUMULEE DES OPERATIONS }\end{array}$} & \multirow{2}{*}{$\begin{array}{c}\text { IMPORTANCE MOYENNE } \\
\text { PAR OPERATION } \\
\text { K€ }\end{array}$} \\
\hline & Nombre & $\%$ & $\mathrm{~K} €$ & $\begin{array}{l}\% \text { du montant } \\
\text { total }\end{array}$ & \\
\hline Collectivité locale & 129 & 30 & 986 & 10 & 7,6 \\
\hline Entreprise privée & 119 & 28 & 7932 & 77 & 65,7 \\
\hline Agriculteur & 82 & 19 & 556 & 5 & 7,3 \\
\hline Association & 58 & 14 & 172 & 2 & 2,9 \\
\hline Gestionnaire d'un réseau & 30 & 7 & 463 & 5 & 15,4 \\
\hline Organisme d'insertion & 11 & 3 & 152 & 1 & 13,9 \\
\hline Total & 429 & 100 & 10261 & 100 & 24,1 \\
\hline
\end{tabular}

A l'échelle des zones étudiées, les collectivités locales représentent les prestataires du plus grand nombre de services environnementaux. Ces services environnementaux restent cependant des opérations de faible ampleur économique. Les communes sont les principaux prestataires agissant en régie directe $(60 \%$ de l'ensemble des services environnementaux produits par des collectivités locales). Ces services en régie directe sont conçus dans le prolongement de leurs interventions traditionnelles. De façon générale, les communes agissent sur des biens-supports dont elles ont la tutelle (espace résidentiel, voirie communale) et produisent des services relativement élémentaires. Les établissements publics de coopération intercommunale, le conseil général et le parc naturel régional, principalement prestataires de services environnementaux auprès des communes, proposent des services environnementaux plus innovants, soit pour leur finalité (pose de franchissements de clôtures dans les parcelles agricoles), soit pour leur bien-support (berges de rivières).

Sur le plan du volume économique, les entreprises privées représentent le premier prestataire à l'échelle des territoires étudiés. $77 \%$ de l'importance économique cumulée des services environnementaux correspond à des commandes auprès d'entreprises privées $(65,7 \mathrm{~K} €$ par opération en moyenne), le plus souvent suite à un appel d'offre d'une collectivité locale. Les entreprises privées sont les principaux intervenants dans les aménagements paysagers de bourg et la restauration de petit patrimoine culturel, car ces activités font appel à une connaissance technique assez précise, et, pour la première, à un capital technique important.

Les agriculteurs constituent logiquement les principaux prestataires de services environnementaux touchant à leurs outils de production (intégration de bâtiments agricoles, 
entretien de parcelles agricoles). Cependant, ils s'impliquent rarement dans d'autres types de services environnementaux, exception faite de la plantation de haies. Les agriculteurs sont parmi les principaux intervenants sur les «points à voir», alors que d'autres catégories de prestataires (associations et communes) agissent de façon plus marquée sur les «points pour voir ». Les agriculteurs produisent dans tous les cas des services environnementaux dotés d'un produit joint, à finalité agricole. Ce résultat souligne la place particulière des agriculteurs dans la fourniture de services environnementaux qui, sans être sur-représentés par rapport aux autres catégories de prestataires, sont nécessairement présents pour certains types de services environnementaux élémentaires, notamment ceux qui font appel au pâturage extensif. Cependant, nous n'avons observé qu'un seul cas où un agriculteur s'est impliqué dans la production de services environnementaux en dehors de son exploitation. Il s'agit d'un agriculteur assurant l'entretien de chemins ruraux pour le compte d'une commune, contre une rémunération annuelle. La rareté de cette situation mérite d'être soulignée.

Les associations interviennent dans $17 \%$ des services environnementaux, bien que le volume économique en reste limité ( $2 \%$ de l'importance économique cumulée des services environnementaux). Cependant les services environnementaux qu'elles produisent ne sont pas négligeables, car ils sont à l'origine de certaines catégories de services environnementaux, repris parfois ultérieurement par d'autres catégories d'intervenants. Par exemple, les associations intervenaient au début des années 1990 dans l'entretien des sentiers de randonnée, ce service ayant ensuite été pris en charge par le conseil général et les communes.

Les gestionnaires d'un réseau (direction départementale de l'équipement, Électricité de France, France Télécom) réalisent des services environnementaux dont l'importance économique moyenne atteint $15,4 \mathrm{~K} €$. Ce sont des opérations relativement importantes, qui correspondent à deux types principaux de services environnementaux élémentaires : l'enfouissement de réseaux et l'aménagement paysager de la voirie. Ils s'impliquent dans les services environnementaux concernant directement les biens-supports dont ils ont traditionnellement la charge.

\section{L'importance et la diversité des circuits institutionnels de financement des services environnementaux complets}

La quasi-totalité des services environnementaux recensés ont fait l'objet d'un financement public (94\% des opérations). $38 \%$ des services environnementaux complets sont uniquement financés par le secteur public local. Leur nombre important traduit leur poids dans l'offre locale de services environnementaux et l'ancrage des services environnementaux dans l'organisation institutionnelle locale. Enfin, les $56 \%$ services environnementaux restants ont bénéficié d'un financement par des politiques publiques, émanant des échelons départemental, régional, national ou européen. Leur poids économique est nettement plus important que les précédents, puisqu'ils représentent $87 \%$ des dépenses totales en faveur des services environnementaux dans les trois zones étudiées.

Quatre grands types de politiques publiques participent au financement du service environnemental. Les politiques agricoles ont subventionné $20 \%$ des opérations réalisées, les politiques de développement $25 \%$, les politiques d'emploi, $10 \%$. Les politiques ayant un objectif principal concernant la protection de la nature n'ont subventionné à titre principal que $2 \%$ des services environnementaux, la faiblesse de ce chiffre est liée au champ de l'environnement retenu. Ces quatre grands types de modalités de financement public des services environnementaux permettent d'esquisser une typologie des services environnementaux selon les secteurs institutionnels au sein desquels ils sont produits: 
l'agriculture, le développement local, l'emploi et la protection de la nature, qui interviennent dans les services environnementaux à dimension collective. Ces secteurs institutionnels, fournissant un cadre spécifique (type de biens-supports, type de prestataires, type de politiques publiques) au service, ne déterminent pas de façon absolue les comportements des agents, mais rendent plus probables certaines relations de services. Par exemple, au sein du secteur institutionnel de l'agriculture, l'espace de référence est en général la parcelle agricole ou l'exploitation dans son ensemble. Le prestataire est quasi-exclusivement un agriculteur et les politiques publiques qui encadrent et financent la relation de services sont portées par les institutions (publiques ou privées) en charge de la gestion de la production agricole.

\section{Du quasi-service au service complet : le cas de la prime à l'herbe}

Nous avons relevé l'ensemble des contrats agri-environnementaux liés au titre de la prime à l'herbe (ou prime au maintien des systèmes d'élevage extensifs), mesure agrienvironnementale instaurée en 1993. Lorsqu'il demande à bénéficier de cette prime, un agriculteur s'engage à entretenir ses prairies et à respecter certaines pratiques de gestion de l'espace, en échange d'une rémunération de $300 \mathrm{~F}(45 €)$ par hectare (à partir de 1995). Une lecture d'économie des services indique que les pouvoirs publics (État et Union européenne) se positionnent comme commanditaire d'un service environnemental d'entretien des prairies, et qu'ils rémunèrent la fourniture de ce service par la prime à l'herbe. Mais, d'après nos observations, il s'agissait incontestablement d'un quasi-service environnemental jusqu'en 1998 car la prime à l'herbe n'induisait alors que peu de contraintes par rapport aux pratiques agronomiques classiques (le coût de production du service restait nul). En 1998, la prime à l'herbe a été réformée, avec des exigences plus fortes concernant l'environnement. Dans certains cas, les agriculteurs ont dû consacrer un temps spécifique au service environnemental, avec un coût de production non nul. Dans ces cas-là, on ne peut plus parler de quasi-service, mais d'une évolution vers un service environnemental complet. Ceci étant, de nombreux agriculteurs bénéficient toujours de la prime à l'herbe, sans intentionnalité spécifique vis-à-vis de l'environnement. D'autres ont adapté leur système agronomique pour continuer à la toucher ou ont choisi de se retirer du dispositif.

Les données concernant la prime à l'herbe collectées pour l'année 2000 montrent que sur les 13 communes étudiées, 395 agriculteurs ont bénéficié de la prime à l'herbe, soit près de 9 agriculteurs sur 10 . Le montant cumulé des versements financiers au titre de la prime à l'herbe en 2000 s'élève à $1005000 €$. En parallèle, le montant total correspondant aux services environnementaux complets produits en 2000 correspond à $1253000 €$ environ. Dans la région où l'étude a été réalisée (région d'élevage de moyenne montagne), la rémunération de l'ensemble des primes à l'herbe est du même ordre de grandeur que le coût de tous les services environnementaux complets recensés.

\section{Conclusion}

Le recours à l'économie des services apporte des éclairages sur certains aspects qui ne sont pas traités par les autres courants économiques qui traitent des services environnementaux. Cette approche conduit à mieux situer la place des agriculteurs dans la fourniture de services environnementaux dans les espaces ruraux, en les comparant à d'autres prestataires de services, et de traiter sur le même plan des prestataires liés explicitement à un support, tel les agriculteurs et les sylviculteurs, et d'autres catégories de prestataires non strictement liés à un 
bien-support. A l'échelle des trois territoires étudiés, les agriculteurs occupent en définitive une place assez réduite dans la fourniture des services environnementaux.

Il y aurait lieu de compléter les observations empiriques par celles d'autres types de services environnementaux, touchant à des biens-supports tels que les eaux souterraines, l'air ou d'autres ressources naturelles, pour voir quel rôle y jouent effectivement les agriculteurs.

Enfin, les catégories intermédiaires entre l'externalité positive de production et le service environnemental complet (quasi-service environnemental passif quasi-service environnemental altruiste) construites et mises en évidence ne sont pas nécessairement des formes stables économiquement. Elles peuvent permettre une lecture dynamique, en termes de trajectoires, de la production des services environnementaux, L'appréhension plus fine de la nature et du volume des externalités positive ou négatives sera alors nécessaire. En effet, les enjeux économiques dont sont porteuses les externalités ne sont pas négligeables, particulièrement dans le domaine de la multifonctionnalité de l'agriculture.

\section{Bibliographie}

Aznar O., Berriet-Solliec M., Guérin M., 2002, "Caractérisation socio-économique de l'intervention publique locale dans la gestion de l'espace rural », Économies et sociétés, $\mathrm{n}^{\circ} 4$, série « économie et gestion des services », 7/2002, p. 1041-1064.

Baumol W.J., Oates W.E., 1988, The theory of environmental policy, New York, Cambridge University Press, $299 \mathrm{p}$.

Berkes F., Folke C., 1992, «A systems perspective ont the inter-relations between natural, human-made and cultural capital », Ecological economics, vol. 5, pp. 1-8.

Bonnieux F., Desaigues B., 1998, Économie et politiques de l'environnement, Paris, Dalloz, $328 \mathrm{p}$.

Bonnieux F., Rainelli P., 2000, «Aménités agricoles et tourisme rural », Revue d'économie régionale et urbaine, $\mathrm{n}^{\circ}$ 5, pp. 803-820.

CNUCED, 1998, Renforcement des capacités des pays en développement dans le secteur des services environnementaux, document de travail, $28 \mathrm{p}$.

Collados C., Duane T.P., 1999, « Natural capital and quality of life : a model for evaluating the sustainability of alternative regional development paths », Ecological economics, vol. 30, n 3, pp. 441-460.

Comolet A., 1994, L'évaluation et la comptabilisation du capital naturel, Paris, éditions l'Harmattan, $258 \mathrm{p}$.

Cornes R., Sandler T., 1996, The theory of externalities, public goods and club goods, New York, Cambridge University Press, 590 p.

Costanza R., d'Argen R., de Groot R., Farber S., Grasso M., Hannon B., Limburg K., Naeem S., O'Neill R.V., Paruelo J., Raskin R.G., Sutton P., van den Belt M., 1997, « The value of the world's ecosystem services and natural capital », Nature, vol. 387, 15 mai, pp. 253-260.

Dabbert S., Dubgaard A., Slangen L., Whitby M., 1998, The economics of landscape and wildlife conservation, CAB International, New York, $286 \mathrm{p}$.

De Bandt J., 1995, Services aux entreprises : informations, produits, richesses, Économica, Paris, $268 \mathrm{p}$.

Delaunay J.-C., Gadrey J., 1987, Les enjeux de la société de service, Paris, Presses de la Fondation nationale des Sciences politiques, $333 \mathrm{p}$.

Delaunay J.-C., 1999, « Capital and other inputs in the production of services - What impact on service relationship ? ", International journal of service industry management, vol. 10, $\mathrm{n}^{\circ} 2$, p. $157-170$. 
Desaigues B., Point P., 1993, Économie du patrimoine naturel : la valorisation des bénéfices de protection de l'environnement, Économica, Paris, $317 \mathrm{p}$.

Desprès A., Normandin D., 1996, «Les services d'environnement fournis par la forêt : évaluation et régulation », Cahiers d'économie et de sociologie rurales, ${ }^{\circ}$ 41, pp. 61-91.

Dorfman R., 1993, "Some concepts from welfare economics » in Dorfman R., Dorfman N. (dir.), Economics of the environment - Selected readings, $3^{\mathrm{e}}$ édition, New York, W. W. Norton \& Company, pp. 79-96.

Gadrey J., 1994, « Les relations de service dans le secteur marchand », in de Bandt J., Gadrey J. (dir.), Relations de services, marchés de services, Paris, éditions du CNRS, pp. 23-41.

Gadrey J., 1996, L'économie des services, $1^{\mathrm{e}}$ éd. 1992, la Découverte, Paris, 126 p.Gadrey J., 2000, "The caracterisation of goods and services : an alternative approach », Review of income and wealth, vol. 46, ${ }^{\circ}$ 3, september, pp. 369-387.

Gillis M., Perkins D., Roemer M., Snodgrass D., 1998, Économie du développement, Paris, Bruxelles, de Boeck université, 784 p.

Hannon B., 1998, « How might nature value man? », Ecological economics, vol. 25, n 3, pp. 265-279.

Hill P., 1977, « On goods and services », Review of income and wealth, vol. 4, n 23, pp. $315-$ 338.

Laurent C., 1994, «L'agriculture paysagiste : du discours aux réalités », Natures sciences sociétés, vol. 2, n 3, pp. 231-242.

Madelin V., 1995, «La rémunération des services environnementaux rendus par l'agriculture », Économie et prévision, n 117-118, pp. 77-88.

Mahé L.-P., 2001, «La multifonctionnalité en quête de légitimité », Problèmes économiques, $\mathrm{n}^{\circ} 2719,27$ juin, pp. 1-3.

Mahé L.-P., Ortalo-Magné F., 2001, Politiques agricoles : un modèle européen, Paris, Presses de la Fondation nationale des Sciences politiques, $235 \mathrm{p}$.

OCDE, 1994, La contribution des aménités au développement rural, Paris, éd. OCDE, 99 p.

OCDE, 2001, Multifonctionnalité - Élaboration d'un cadre analytique, Paris, éd. OCDE, $177 \mathrm{p}$.

OMC, 2000, Communication des Communautés européennes et de leurs États membres AGCS 2000 : Services concernant l'environnement, Genève, Suisse, $10 \mathrm{p}$.

Willinger M., 1996, « La méthode d'évaluation contingente : de l'observation à la construction des valeurs de préservation », Natures, sciences, sociétés, vol. 4, n 1 , janvier, p. 6-22.

\section{Résumé :}

Ce texte discute de la notion de «service environnemental» en rapport avec la multifonctionnalité de l'agriculture et des campagnes. Les auteurs présentent les trois principales conceptions de cette notion dans la littérature économique : selon l'économie écologique, l'économie de l'environnement et l'économie des services. Ensuite ils proposent un cadre d'analyse des services environnementaux à partir de l'économie des services. Enfin, la partie empirique repose sur un inventaire de ces services dans trois territoires ruraux.

\section{Summary :}

This paper discusses the term 'environmental service', about the multiple functions of farming and of the countryside. The authors set out the three main conceptions of environmental services in the economic literature, by reference to ecological economics, 
environmental economics and service economics. Then they propose a analytical framework of environmental services derived from service economics. Finally, an empirical study is based on an inventory of environmental services produced in three rural areas.

Mots-clés : Services ; espace rural ; environnement ; biens publics ; collectivités locales. Services ; rural areas ; environment ; publics goods ; local authorities

JEL : R0 ; R14 ; Q2 ; Q26 